\title{
Electrothermal Feedback in Polypyrrole Coated Cotton Fabric
}

\author{
Abbasi A. M. Rehan ${ }^{\mathrm{a}, \mathrm{b}, *}$, RAmadAn Mohamed A. ${ }^{\mathrm{a}, \mathrm{c}}$, WIENER Jakub $^{\mathrm{a}}$, Baheti Vijay ${ }^{\mathrm{a}}$ \\ MiLiTKY Jiri ${ }^{a}$ \\ a Faculty of Textile Engineering, Technical University, Studentska 2, 46117, Liberec, Czech Republic \\ ${ }^{\mathrm{b}}$ Department of textile engineering, BUITEMS, Takatu Campus, 87100, Quetta, Pakistan \\ ${ }^{\mathrm{c}}$ Textile Research Division, National Research Center, 12311, Cairo, Egypt
}

Received 7 January 2013; accepted for publication 25 March 2013

\begin{abstract}
In this study, polypyrrole (PPy) was coated by chemical oxidation through in-situ polymerization technique. Tetraethyl ammonium p-toluene sulfonate (TEAp-TS) was chosen as a doping agent along with $\mathrm{FeCl}_{3}$ as oxidizing agent. Six different fabric samples were prepared by varying concentrations of monomer, doping agent and oxidizing agent in order to obtain samples with different electrical resistivity. Each sample was exposed to four different temperatures $\left(80,120,160\right.$ and $\left.200{ }^{\circ} \mathrm{C}\right)$ and electrical resistivity was recorded at different intervals in the range 0-600 seconds. In this way the influence of temperature on electrical resistivity of PPy coated cotton fabric samples was analyzed and found that resistivity decreases with the increase in temperature in two minutes significantly. FT-IR spectrum confirms that there is no change in PPy before and after heating of samples. SEM micrographs showed the deposition of PPy on cotton fibers surface.
\end{abstract}

Key Words: Cotton fabric, Polypyrrole, Thermal influence

\section{Introduction}

In recent time, most scientific researchers turned to prepare conductive substrates by inserting metal into insulating resin or coating a plastic substrate with a conductive metal solution [1]. In this way, conductive fabrics can be prepared to obtain conductive textiles which are very interesting materials because they generally have application in different areas. The electrically conductive textile materials can be obtained when treated with intrinsically conducting polymers (ICPs) which have received the considerable attention of scientists because of their good environmental stability and their application in different areas such as manufacturing of organic transistors and light-emitting diodes [2], functional textiles $[3,4]$, organic electrodes $[5,6]$, coatings for fuel cells, corrosion protection [7,8] and biosensors [9]. The most prominent ICPs are polypyrrole and polyaniline, where electrical conductivity can have values comparable to those observed for poorly conducting metals and alloys [10]. These textile materials are even able to absorb as well as reflect electromagnetic waves also [11]. They have also been proposed and tested for acidity-sensing, heating devices [12], design of actuators $[13,14]$, flame retardation $[15,16]$ and for printing of conducting patterns on paper [17], therefore they can exhibit certain advantages over metallic materials. Furthermore, the conductive fabrics are one of the most important of smart/technical materials [18]. ICPs are conjugated polymers, with alternating single and double bonds in the polymer backbone which is a necessary condition for charge carriers to move freely along the polymeric chain when doping is provided.

Pyrrole can be polymerized by electrochemical oxidation on metallic substrates or by chemical oxidation, and its conducting properties, in terms of conductivity and environmental stability, can be adjusted by doping the polymer with anionic surfactants [19]. Furthermore, some polypyrrole properties (and those of ICPs in general) i.e. volume, surface energy and ionic conductivity depend on the polymer redox state. Polypyrrole was polymerized on the surface of cellulose fibres using a sequence of fiber impregnation in $\mathrm{FeCl}_{3}$ solutions, thickening and re-dispersion in a pyrrole solution [20]. The conductive cotton fabrics prepared by polymerization of pyrrole in the presence of $\mathrm{FeCl}_{3}$ as an oxidizing agent and TEAp-TS a doping agent which in turn caused a reduction in the moisture regain of the substrate, resulted in production of conductive cotton fabrics in laboratory scale.

Our research aimed to prepare cotton fabric samples having different levels of electrical resistivity and to study the influence of temperature on the resistivity of the samples.

\footnotetext{
* Corresponding author: E-mail: rehan.abbasi@tul.cz, Tel: +420-734-823-366
} 


\section{Methodology}

\subsection{Materials and sample preparation}

Bleached cotton fabric was obtained from INOTEX in Czech Republic. Pyrrole monomer was obtained from Merck and Iron (III) Chloride of analytical grade from BDH Chemicals was used. TEAp-TS $\left[\left(\mathrm{C}_{2} \mathrm{H}_{5}\right)_{4} \mathrm{~N}\left(\mathrm{CH}_{3} \mathrm{C}_{6} \mathrm{H}_{4} \mathrm{SO}_{3}\right)\right]$ was obtained from Aldrich Chemicals. All the chemicals were used as received.

PPy was coated on the cotton fabric by in-situ polymerization technique by the following steps. In the first step, an aqueous solution of $\mathrm{FeCl}_{3}$ and TEAp-TS was prepared by molar ratio 1:2, then the cotton fabric sample was added to the solution by keeping the liquor ratio $1: 25$, for $20 \mathrm{~min}$. The fabric sample was then passed through pneumatically controlled horizontal padders with $70 \%$ pick up.

The padded fabric sample was then introduced into Ahiba Nuance dye pots which contain aqueous dispersion of pyrrole by keeping the liquor ratio 1:25. Pots were then placed into a rapid rotary dyer for 4 hours under ambient conditions. Agitation was carried out via rotation of the pots at 25 revolutions per min. Direction of rotation was changed automatically in each after 1 minute. After polymerization the samples were then thoroughly washed with a sufficient amount of distilled water several times to remove the excess of monomer and bi-products and allowed to dry in the air at ambient conditions. Six samples of different electrical resistivity were prepared by taking different concentrations of pyrrole, $\mathrm{FeCl} 3$ and TEAp-TS, as described in the Table 1.

\subsection{Testing and analysis}

The electrical resistivity of the PPy-coated cotton fabric was measured using the AATCC 76 two point-probe technique using

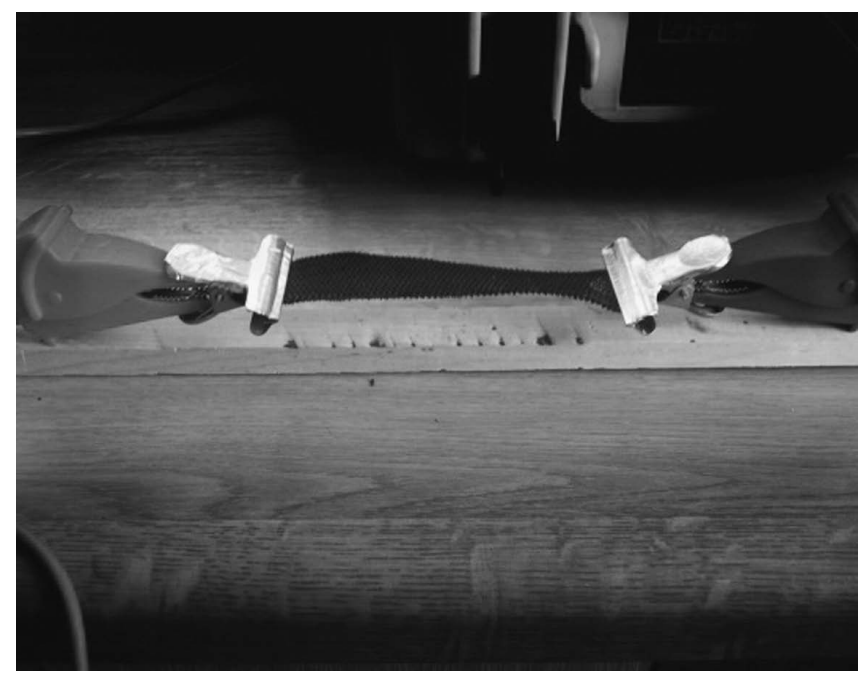

(a) Electrodes mounted on wooden stick.
Table 1 Recipes for the preparation of substrates.

\begin{tabular}{cccc}
\hline Substrate label & $\begin{array}{c}\text { Pyrrole conc. } \\
{[\mathrm{mol} / \mathrm{l}]}\end{array}$ & $\begin{array}{c}\mathrm{FeCl}_{3} \text { conc. } \\
{[\mathrm{mol} / \mathrm{l}]}\end{array}$ & $\begin{array}{c}\text { TEAp-TS conc. } \\
{[\mathrm{mol} / \mathrm{l}]}\end{array}$ \\
\hline $\mathrm{A}$ & 0.2 & 0.1 & 0.05 \\
\hline $\mathrm{B}$ & 0.2 & 0.15 & 0.075 \\
\hline $\mathrm{C}$ & 0.2 & 0.2 & 0.1 \\
\hline $\mathrm{D}$ & 0.2 & 0.25 & 0.125 \\
\hline $\mathrm{E}$ & 0.1 & 0.25 & 0.125 \\
\hline $\mathrm{F}$ & 0.3 & 0.25 & 0.125 \\
\hline
\end{tabular}

FLUKE digital multimeter (uni-T, UT70C). The bulk electrical resistance of the dried PPy coated fabric samples was measured in the heating oven (Venticell) at different temperatures and different time intervals. In order to measure electrical resistance of the sample during heating, cotton sample was clamped between clamps as electrodes having electrical resistivity of $1.3 \times 10^{-4} \Omega . \mathrm{cm}$ as shown in figure 1 (a). Figure 1 (b) shows the heating oven with a narrow hole in the side wall to mount the sample along with measuring electrodes. High temperature resistant rubber seal was placed around the electrode to prevent the heat loses.

The surface morphology of PPy coated cotton fabric was investigated by using SEM, TESCAN VEGA USA Inc. in Technical University of Liberec. Before SEM imaging, the fabric samples were coated with gold particles.

The FT-IR spectra of cotton fabrics treated with polypyrrole were recorded on a FT-IR spectrometer by Perkin-Elmer Inc, in the spectral range 4000-400 cm-1 using the $\mathrm{KBr}$ disc technique.

Tensile strength of the substrates was measured by Universal Electromechanical Testing Machine LabTest ${ }^{\circledR} \mathrm{S}$ manufactured by LaborTech ${ }^{\circledR}$ in Czech Republic. The machine was placed in the Technical University of Liberec, Czech Republic which runs on

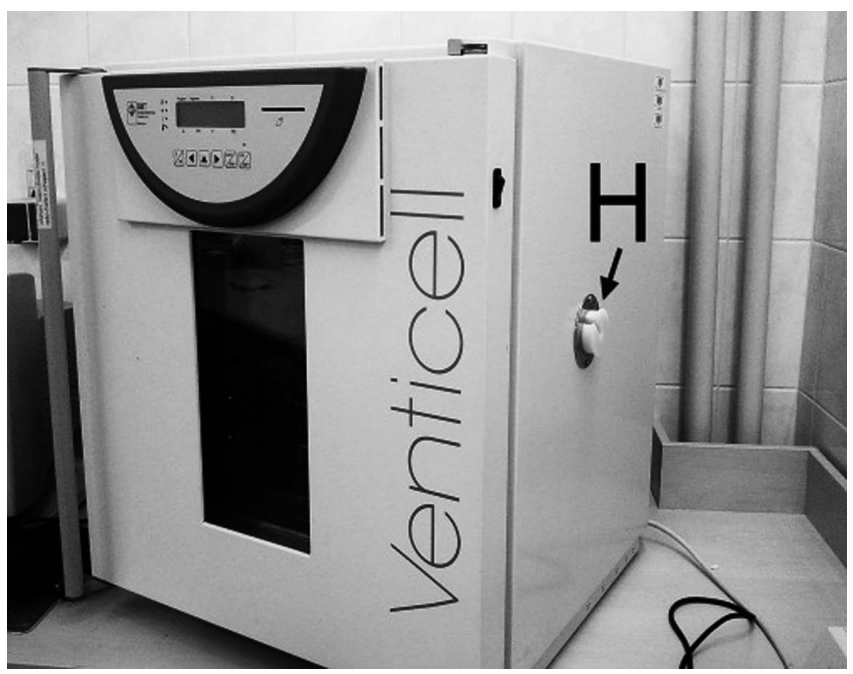

(b) Heating oven with the hole, " $\mathrm{H}$ " in the side wall.

Fig. 1 Equipment for the experiments. 
the software Test \& Motion ${ }^{\circledR}$ and strength calibrated according to EN ISO 7500-1 and displacement calibrated according to EN ISO 9513.

\section{Results and discussion}

\subsection{Sample preparation and measurement of electrical resistivity}

Six samples of cotton fabric were coated by PPy through chemical oxidative polymerization of pyrrole monomer in the presence of TEAp-TS and $\mathrm{FeCl}_{3}$ and their behaviors under dry thermal heating were recorded.

Oxidant salts such as ferric chloride $\left(\mathrm{FeCl}_{3}\right)$ function as both the oxidant and the doping agent, and hence the polymer is obtained in the electrically conducting form. Other transition metal salts can also be used as oxidizing agents for the polymerization of pyrrole. The transition metal ion is an electron acceptor. Therefore it oxidizes the $\pi$-electron system of the pyrrole ring at the initiation step. The intermediate steps of the polymerization of pyrrole initiated by oxidant to yield a radical cation are as follows [21]:<smiles>Cc1ccc[nH]1</smiles>

Radical cations recombine to form dications:<smiles>CC1CC=C[NH+]1Cc1ccc[nH]1</smiles>

Deprotonation of dication to yield a dimer:<smiles>CC#[PH+]C</smiles>

The above process continues by oxidation of the dimer to yield a radical cation of the dimer and the combination of radical cations to form trimers, tetramers, and polymers.

The electrical resistivity of all the substrates were measured at room temperature $24 \pm 1{ }^{\circ} \mathrm{C}$ as well as at different temperatures $\left(80,120,160\right.$ and $\left.200^{\circ} \mathrm{C}\right)$ at different time intervals from 0-600 seconds and but data points presented in figures 4 and 5 are plotted for 120 and 600 seconds. In all the plots, each measurement of electrical resistivity was converted into normalized value in order to minimize the variation in the data points of different series and plotted against temperature range.

Increase in concentration of the oxidizing agent and doping agent on the fabric substrate and keeping pyrrole monomer concentration constant, ultimately increases the PPy concentration on substrate which causes significant decrease in electrical resistivity as shown in figure 2. Similar effect can be seen in figure 3 where concentrations of $\mathrm{FeCl}_{3}$ and TEAp-TS were kept constant

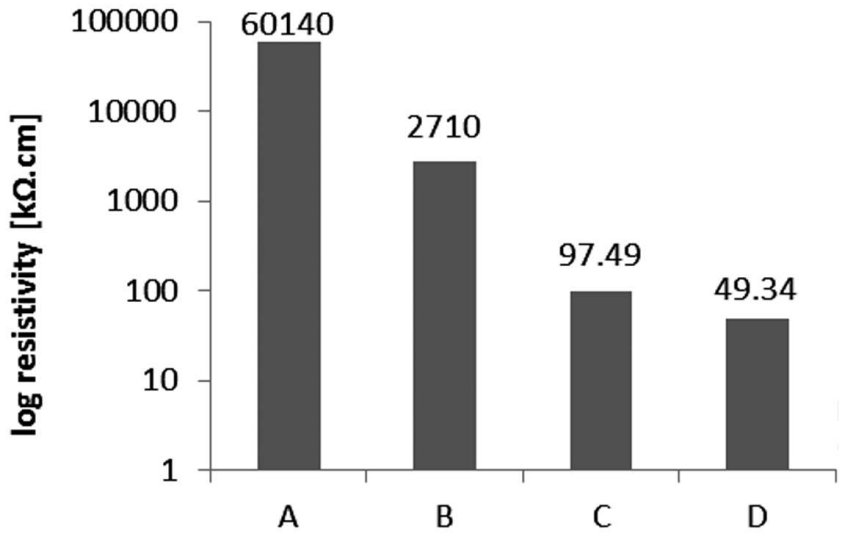

Fig. 2 Effect of concentration of oxidant and dopant on resistivity of PPy coated cotton fabric substrates.

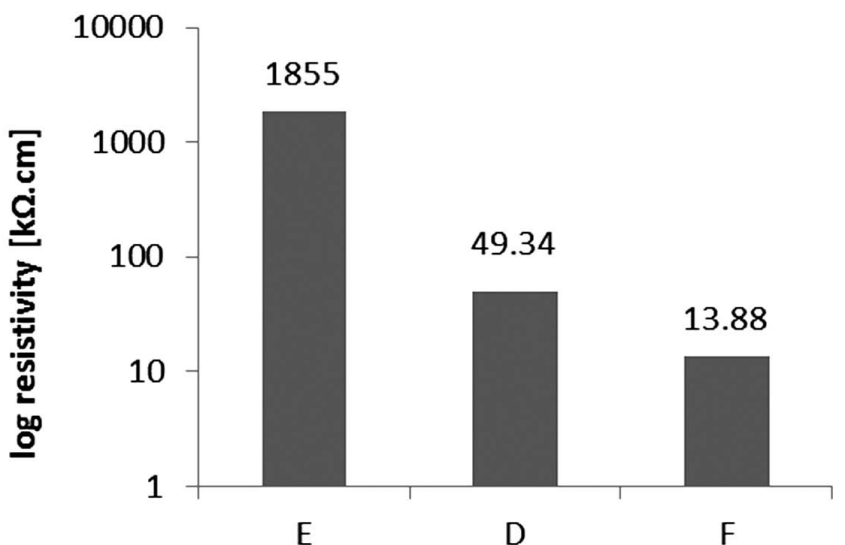

Fig. 3 Effect of concentration of pyrrole on resistivity of PPy coated cotton fabric substrates.

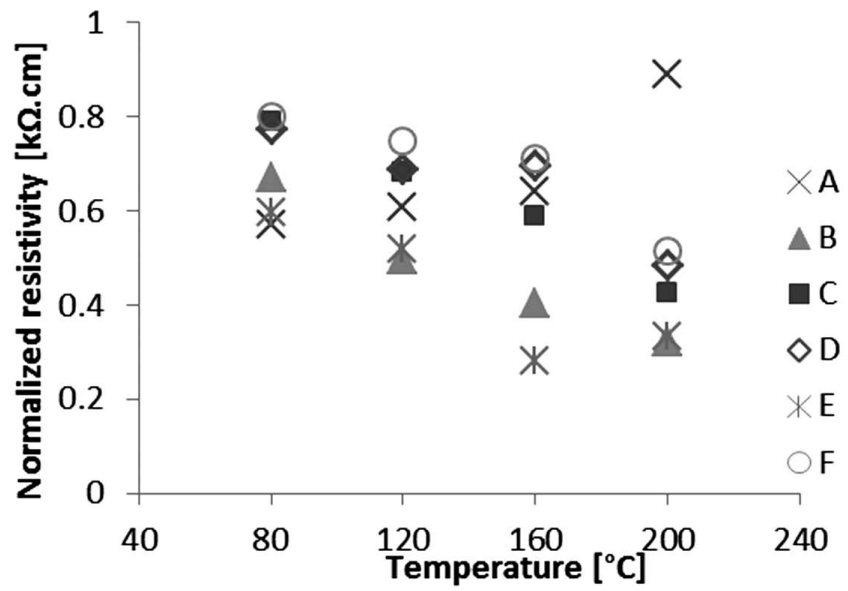

Fig. 4 Effect of temperature on resistivity of PPy coated cotton fabric substrates after 120 seconds. 


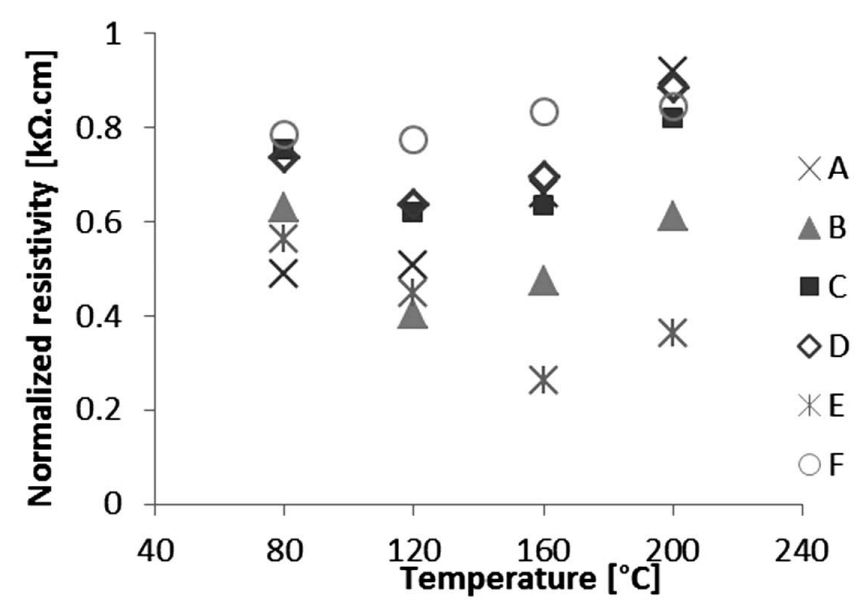

Fig. 5 Effect of temperature on resistivity of PPy coated cotton fabric substrates after 600 seconds.

and concentration of pyrrole monomer was increased and substantial drop in electrical resistivity was observed.

All the six samples were introduced to the heating oven one by one at the four different temperatures as mentioned earlier and the data points collected after 120 and 600 seconds were plotted in figure 4 and figure 5 respectively. In figure 4 it can be perceived that all the samples lose their electrical resistivity upon heating at all the temperatures in 120 seconds except sample "A" that have the highest electrical resistivity among all the six samples. The decline in resistivity of the samples is very drastic for this time period; this behavior is in the close agreement to the general behavior of semiconductors [22].

Conductivity of a material is determined by two factors: the concentration of free carriers available to conduct current and their mobility (or freedom to move). In a semiconductor, both mobility and carrier concentration are temperature dependent. The mobility of the carriers in a semiconductor is influenced by the presence of charged impurities. Impurity scattering is caused by crystal defects such as ionized impurities. At lower temperatures, carriers move more slowly, so there is more time for them to interact with charged impurities. As a result, as the temperature decreases, impurity scattering increases and the mobility decreases.

In this research, with the passage of time the cotton fabric sample attains the temperature of the surrounding environment and from that point lattice scattering increases which ultimately decreases the mobility of free carriers that can be seen in figure 5 .

The electrical resistivity $\rho$ of most materials changes with temperature $\mathrm{T}$. If the temperature $\mathrm{T}$ does not vary too much, a linear approximation is typically used:

$$
\rho(\mathrm{T})=\rho_{\mathrm{o}}\left[1+\alpha\left(\mathrm{T}-\mathrm{T}_{\mathrm{o}}\right)\right]
$$

where $\alpha$ is called the temperature coefficient of resistivity in $\left[\mathrm{K}^{-1}\right]$, To is a fixed reference temperature in $[\mathrm{K}]$ (usually room temperature) and $\rho_{\mathrm{o}}$ is the resistivity at temperature To. The parameter $\alpha$ in equation 1 is an empirical parameter fitted from

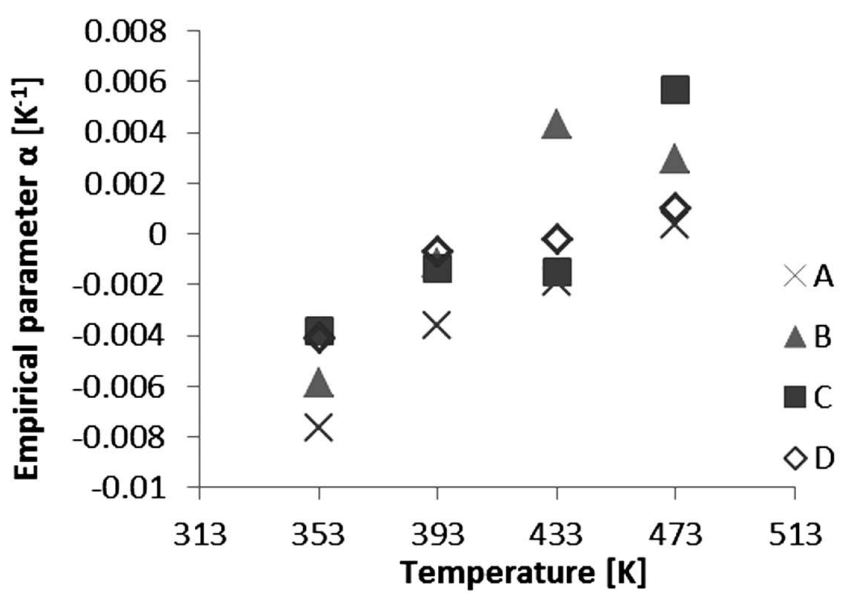

Fig. 6 Empirical parameter a calculated on different temperatures for substrates A, B, C and D.

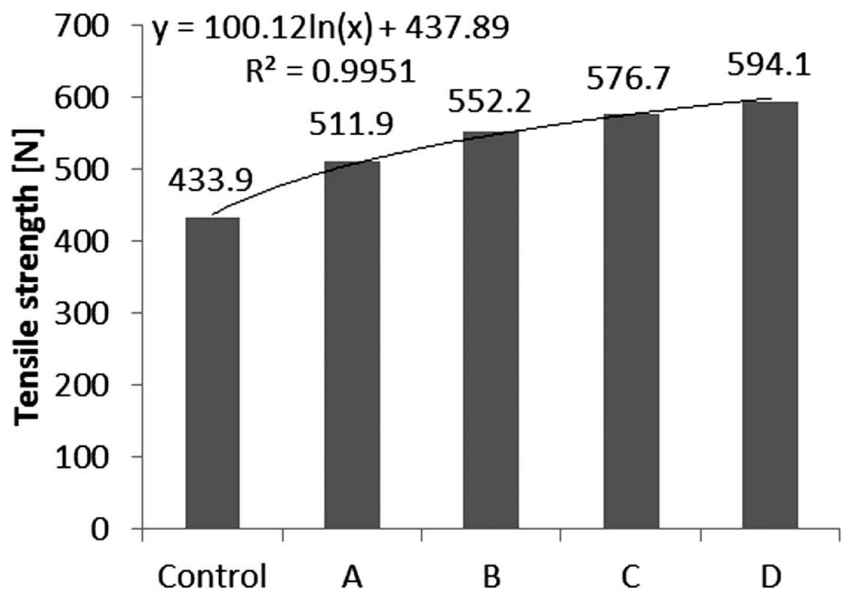

Fig. 7 Effect of concentration of oxidant and dopant on tensile strength of PPy coated cotton fabric substrates.

measurement data. Because the linear approximation is only an approximation, $\alpha$ is different for different reference temperatures and in this study it changes linearly with the change in temperature as shown in figure 6. Negative values of $\alpha$ show the typical behavior of semiconductors whereas the positive temperature coefficient (PTC) refers to the material that experiences an increase in electrical resistance with increase in their temperature such as conductors [23].

The tensile strength of control and four PPy coated fabric substrates A, B, C and D were tested by ISO 13934-1 and breaking strength were plotted in figure 7 for comparison. Here the improvement in tensile strength of the cotton fabric after coating with PPy in different concentrations can be observed. Concentration was increased from substrate "A" to " $D$ " as mentioned in Table 1. During in-situ polymerization PPy covers each individual fiber and ultimately increase the overall mechanical properties of the substrate. Increase in tensile strength follows logarithmic function with $\mathrm{R}^{2}=0.9951$. 


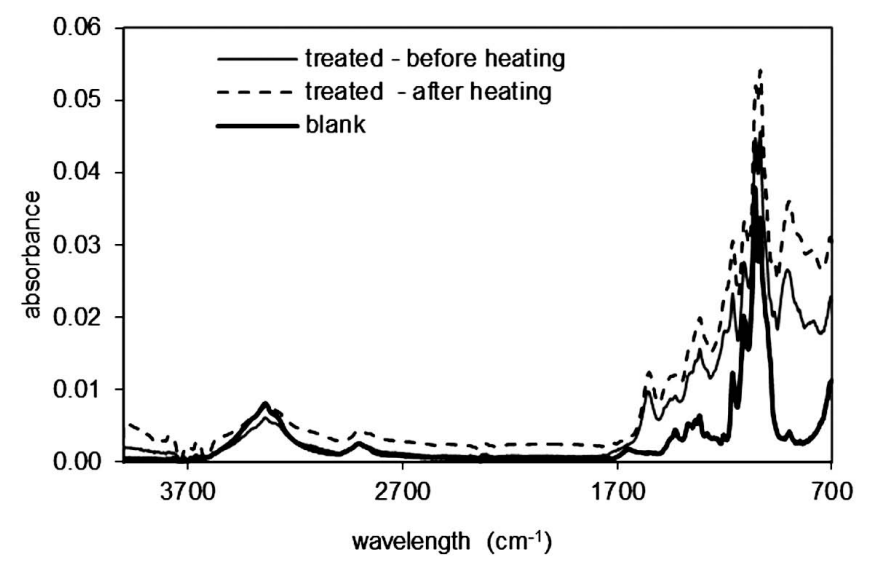

Fig. 8 FT-IR spectra of PPy coated sample (before and after heating) and uncoated cotton fabric sample.

\subsection{FT-IR analysis}

The FT-IR spectrum of the treated cotton fabric before and after heating, as shown in figure 8 reveals the presence of a broad band at $3300-3500 \mathrm{~cm}^{-1}$ attributable to the $\mathrm{OH}$ groups, a strong absorption band at about $1566 \mathrm{~cm}^{-1}$ which is assigned to the $\mathrm{C}=\mathrm{N}$ and $\mathrm{C}=\mathrm{C}$ groups, with notice that this absorption band disappeared in the case of untreated cotton fabric. This FT-IR spectrum confirms the presence of polypyrrole in the treated cotton fabric before and after thermal treatment and there is no significant difference between them.

\subsection{SEM analysis}

The morphology of cotton fibers after deposition of PPy was examined by SEM images. It can be observed from figure 9 ( $a, b$ and c) that in this method of polymerization submicron circular particles are produced which get aggregated with the passage of time and deposit on the fiber surface.

\section{Conclusion}

A few different samples of cotton fabric were coated through insitu polymerization of pyrrole monomer in the presence of $\mathrm{FeCl}_{3}$ and TEAp-TS by varying their concentrations. The influence of dry thermal treatment of coated fabric samples at different temperatures on electrical resistivity at different time intervals was studied. It was found that electrical resistivity of coated fabric samples decreases with an increase in temperature significantly till 120 seconds. It is also worth noticing that electrical resistivity decreases drastically when a sample is subjected to particular temperature but at a time when a sample attains the temperature of the environment, the drop in resistivity is no more noteworthy. At temperatures 80 and $120^{\circ} \mathrm{C}$ loss in resistivity is stabilized whereas at high temperatures i.e. at 160 and $200^{\circ} \mathrm{C}$ electrical resistivity reflected back to increase. This phenomenon is more pronounced with low resistive or highly conductive samples.

IR spectroscopy confirms the presence of PPy on the cotton fabric substrate and that dry thermal treatment of these samples causes no influence on the structure of PPy.

\section{Acknowledgment}

This research work was financed by Student Grant Scheme (SGS) in the Technical University of Liberec, Czech Republic.

\section{References}

[1] Edenbaum J (1992) "Plastics Additives and Modifiers Handbook", p98, Van Nostrand Reinhold, New York

[2] Carpi F, De Rossi D (2006) Optics Laser Tech, 38, 292-305

[3] Dall' Acqua L, Tonin C, Peila R, Ferrero F, Catellani M (2004) Synth Met, 146, 213-221

[4] Lin T, Wang L, Wang X, Kayan A (2005) Thin Solid Films, 479, $77-82$

[5] Otero TF, Cantero I (1999) Power Sources, 81- 82, 838-841

[6] Schultze JW, Karabulut H (2005) Electrochim Acta, 50,

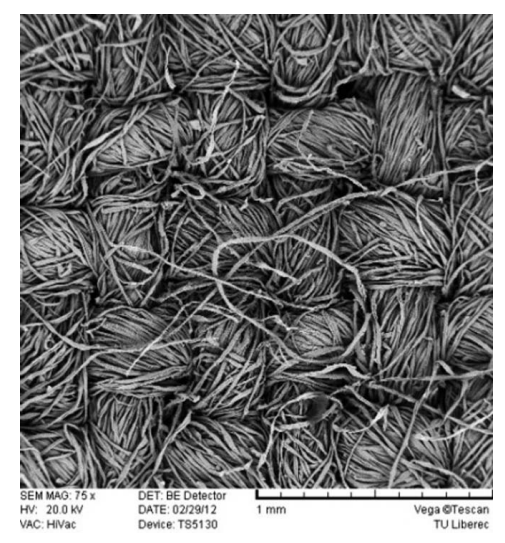

(a)

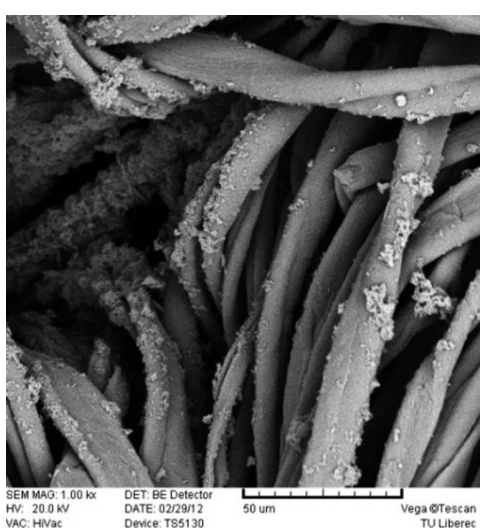

(b)

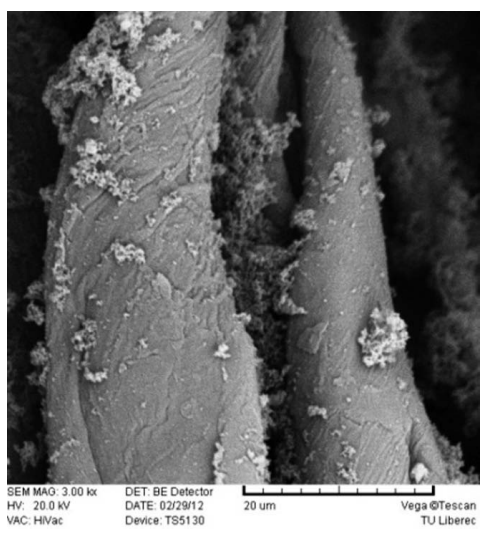

(c)

Fig. 9 SEM micrographs of PPy coated cotton fabric sample. 
$1739-1745$

[7] Bouzek K, Mangold KM, Juttner K (2001) Electrochim Acta, 46, 661-670

[8] Breslin CB, Fenelon AM, Conroy KG (2005) J Mater \& Des, 26, 233-237

[9] Gerard M, Chaubey A, Malhotra BD (2002) Biosens Bioelectron, 17, 345-359

[10] Avloni J, Ouyang M, Florio L, Henn AR, Sparavigna A (2007) Thermoplas Compos Mater, 20, 241

[11] Sapurina I, Kazantseva NE, Ryvkina NG, Prokeš J, Sha P, Stejskal J (2005) Appl Polym Sci, 95, 807-814

[12] Bhat NV, Seshadri DT, Nate MM, Gore AV (2006) Appl Polym Sci, 102, 4690-4695

[13] Kim J, Deshpande SD, Yun S, Li Q (2006) Polymer J, 38, 659-668

[14] Deshpande SD, Kim J, Yun SR (2005) Smart Mater Struct, 14, 876-880

[15] Stejskal J, Trchov M, Sapurina I (2005) Appl Polym Sci, 98,
2347-2354

[16] Stejskal J, Trchov M, Brodinov J, Sapurina I (2007) Appl Polym Sci, 103, 24-30

[17] de Barros RA, Martins CR, de Azevedo WM (2005) Synth Met, 155, 35-38

[18] Abbasi AMR, Mangat MM, Baheti V, Militky J (2012) J Fib Bioeng Inf, 5, 163-168

[19] Omastova M, Mičušik M (2012) Chem Pap, 66, 392-414

[20] Beneventi D, Nortier P, Alila S, Boufi S, Chaussy D (2006) Cellulose, 13, 725-734

[21] Rodriguez J, Grande HJ, Otero TF (1997) "Handbook of Organic Conductive Molecules and Polymers", 2, p415, John Wiley and Sons, New York

[22] Kasap SO (2005) "Principles of Electronic Materials and Devices”, 3, p374, McGraw-Hill, New York

[23] Alenitsyn AG, ButikovEI, Kondratyev AS (1997) "Concise Handbook of Mathematics and Physics", pp331-332, CRC Press, New York 